\title{
Comparative Aspects of Spin-Dependent Interaction Potentials for Spin-1/2 and Spin-1 Matter Fields
}

\author{
P. C. Malta, ${ }^{1,2}$ L. P. R. Ospedal, ${ }^{2}$ K. Veiga, ${ }^{2,3}$ and J. A. Helayël-Neto ${ }^{2}$ \\ ${ }^{1}$ Institut für Theoretische Physik, University of Heidelberg, Philosophenweg 16, 69120 Heidelberg, Germany \\ ${ }^{2}$ Centro Brasileiro de Pesquisas Físicas (CBPF), Rua Dr. Xavier Sigaud 150, Urca, 22290-180 Rio de Janeiro, RJ, Brazil \\ ${ }^{3}$ Instituto Federal de Educação, Ciência e Tecnologia da Bahia, Campus Vitória da Conquista, Avenida Amazonas 3150, Zabelê, \\ 45075-265 Vitória da Conquista, BA, Brazil
}

Correspondence should be addressed to P. C. Malta; malta@thphys.uni-heidelberg.de

Received 14 December 2015; Revised 4 February 2016; Accepted 17 February 2016

Academic Editor: Frank Filthaut

Copyright (C) 2016 P. C. Malta et al. This is an open access article distributed under the Creative Commons Attribution License, which permits unrestricted use, distribution, and reproduction in any medium, provided the original work is properly cited. The publication of this article was funded by $\mathrm{SCOAP}^{3}$.

\begin{abstract}
This paper sets out to establish a comparative study between classes of spin- and velocity-dependent potentials for spin-1/2 and spin-1 matter currents/sources in the nonrelativistic regime. Both (neutral massive) scalar and vector particles are considered to mediate the interactions between (pseudo-)scalar sources or (pseudo-)vector currents. Though our discussion is more general, we contemplate specific cases in which our results may describe the electromagnetic interaction with a massive (Proca-type) photon exchanged between two spin-1/2 or two spin-1 carriers. We highlight the similarities and peculiarities of the potentials for the two different types of charged matter and also focus our attention on the comparison between the particular aspects of two different field representations for spin-1 matter particles. We believe that our results may contribute to a further discussion of the relation between charge, spin, and extensibility of elementary particles.
\end{abstract}

\section{Introduction}

Back to 1950, the paper by Matthews and Salam [1] and the subsequent works by Salam in $1952[2,3]$ clarified the quantum field-theoretical approach to the electrodynamics of (massive) charged scalar particles. Ever since, the problem of extending this investigation to include the case of (massive) charged vector particles became mandatory in view of the theoretical evidence for the role of charged and neutral spin-1 particles that should couple to the charged and neutral currents.

From 1960, Komar and Salam [4], Salam [5, 6], Lee and Yang [7], and Salam and Delbourgo [8] gave a highly remarkable push for the understanding and construction of a fundamental theory for the microscopic interaction between charged vector bosons and photons. Further works by Tzou [9], Aronson [10], and Velo and Zwanzinger [11] summed up efforts to the previous papers and the final conclusion was that a consistent unitary and renormalizable quantum fieldtheoretical model would be possible only in a non-Abelian scenario with a Higgs sector that spontaneously breaks the gauge symmetry to give mass to the vector bosons, without spoiling the unitarity bounds for the cross section of the scattering of longitudinally polarized charged vector bosons [12].

Here, we adopt this framework: the electrodynamics of (massive) charged vector bosons with a nonminimal dipoletype term coupling to the gauge field. In the case of the massless photon, this ensures a $g=2$-value for the $g$-ratio of spin-1 particles. We should however point out that this dipole-type interaction is nonminimal from the viewpoint of an Abelian symmetry; if we take into account the local $S U(2)$ symmetry that backs the plus- and minus-charged vector bosons, the dipole coupling in the action is actually a minimal (nonAbelian, $S U(2)$ ) interaction term after spontaneous symmetry breaking has taken place.

In the present paper, our main effort consists in pursuing a detailed investigation of the semiclassical aspects of the charge and spin interactions for massive charged matter of a vector nature. Our central purpose is to compare the features 
and specific profiles of the influence of the spin of the charge carriers on interaction potentials (electromagnetic or a more general $U(1)$ interaction) between two different categories of sources/currents: fermionic and spin-1 bosonic. We will present the conclusions of our comparative procedure at the end of our calculations.

At this point, we highlight that the literature in the topic contemplates a great deal of articles discussing the structure of the electromagnetic current and electrodynamical aspects of spin-1 charged matter [13-23]. We pursue an investigation of an issue not considered in connection with spin-1 charged matter: the spin- and velocity-dependence of the interaction potential associated with (pseudo-)scalar sources and pseudo-vector currents that interact by exchanging scalar and vector mediators, respectively. For the spin-1, these specific cases have not been addressed to in the literature. These extra sources/currents may not necessarily be associated with the electromagnetic interaction in that they do not follow from the $U(1)$ symmetry of the electromagnetism. We may be describing a new force between these extra sources/current whose origin could be traced back to some more fundamental physics. The case of the usual vector current is reassessed here and our results match with the ones in the literature. It is worth mentioning that fermionic sources/currents can display a wide range of spin- and velocity-dependent interparticle potentials [24-26], and several of them have been reconsidered in this paper.

The electrodynamics of ordinary fermionic matter is very well understood, from the macroscopic to the quantum level. Scalar and vector bosonic charged matter, on the other hand, experience a richer variety of interactions when coupled to the electromagnetic field. So far, most of the theoretical and experimental literature dealing with macroscopic interactions consider only spin-1/2 matter, that is, fermionic sources/currents [27-30]. This preference is naturally due to the fact that ordinary charges in matter are carried by electrons. Elementary spin-1 charged particles are difficult to observe, since the only known examples are the $W^{ \pm}$gauge bosons, whose mass is too large, and lifetime too short, to allow direct inspection (its full width is $\Gamma_{W} \sim 2 \mathrm{GeV}$ [31]). Though not stable enough to be directly handled, elementary spin-1 particles have, in principle, their own electrodynamics and it is of theoretical interest to study how they deviate, or not, from their fermionic counterpart. On the other hand, at the atomic and nuclear level, it would be a good motivation to have a spin- and velocity-dependent expression for the electromagnetic potential between ionised spin-1 $($ charge $= \pm 1)$ atoms and charged spin-1 nuclei or hypernuclei $[32,33]$.

We highlight here a particular feature of bosonic charge carriers as far as the electromagnetic interactions are concerned. From a purely macroscopic point of view, the Maxwell equations address the problem of determining field configurations from given charge and current densities and a number of duly specified boundary conditions. They do not take into account the microscopic nature of these sources ( $\rho$ and $\vec{j}$, resp.). If a microscopic description of charged matter in terms of classical fields is given (based on a local $U(1)$ symmetry), the particular aspects of the charge carriers become salient and London-type terms [34] may arise. We

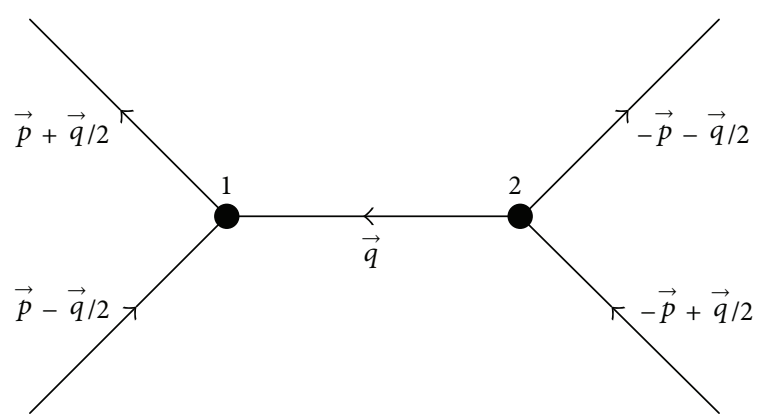

Figure 1: Momenta assignments for the tree-level interaction between sources/currents 1 and 2 .

will be more specific about this point at the final section of our paper, where we render more evident the peculiarities of the spin of the charge carriers in the electromagnetic and general Abelian interactions.

Our paper is outlined as follows: in Section 2, we introduce the concept of potential and briefly discuss the kinematics involved, as well as presenting the notations and conventions employed. In Section 3, we calculate the sources/currents and potentials, discussing the similarities between spin-1/2 and spin-1 matters. We consider, in Section 4, another possible field representation for the spin-1 charged carrier, namely, via a rank-2 tensor field, and we also work out the corresponding source/current-source/current interaction potentials. We present our conclusions and perspectives in Section 5. Natural units are adopted throughout, where $\hbar=c=1$, and we assume $\epsilon^{0123}=+1$.

\section{Methodology}

Since we are interested in comparing the low-energy behavior of spin-1/2 and spin- 1 as the interacting matter sector, it is convenient to work in the nonrelativistic (NR) limit of the interactions. For simplicity we choose to work in the center of mass (CM) reference frame, in which particle 1 has initial and final 3-momenta given by $\vec{P}=\vec{p}-\vec{q} / 2$ and $\vec{P}^{\prime}=\vec{p}+\vec{q} / 2$, respectively. Here, $\vec{p}$ is the average momentum of particle 1 before and after interaction, while $\vec{q}$ is the momentum transfer carried out by the intermediate boson; see Figure 1 .

The potential is calculated through the first Born approximation [35], that is, the Fourier transform of the amplitude, $\mathscr{A}(\vec{q}, m \vec{v})$, with respect to the momentum transfer:

$$
V(r)=-\int \frac{d^{3} \vec{q}}{(2 \pi)^{3}} \mathscr{A}(\vec{q}, \vec{p}) e^{i \vec{q} \cdot \vec{r}}
$$

where we explicitly assume that the amplitude contains terms dependent on the velocity of particle 1 . It is clear from the NR approximations of the sources/currents presented below that not only velocity-dependent terms but also spin-dependent ones will arise in the potentials. This is due to our choice of keeping terms in the currents which go beyond the zerothorder, thus being static, case [36].

In what follows, we will consider only elastic interactions, which, together with energy conservation, imply $q^{0}=0$ and 
$\vec{p} \cdot \vec{q}=0$, pointing out that $q$ is a space-like 4-vector: $q^{2}=-\vec{q}^{2}$. Furthermore, in the amplitude we will keep only terms up to second order in $|\vec{p}| / m_{1,2}$.

The Feynman rules for the tree-level diagram above are equivalent to taking $i J_{1,2}$ as the interaction vertices, where $J_{1,2}$ are the matter sources/currents associated with particles 1 and 2, respectively. The corresponding amplitude may then be written as

$$
\mathscr{A}=i J_{1}\langle\text { prop. }\rangle J_{2},
$$

where $\langle$ prop. $\rangle$ is the momentum-space propagator of the intermediate boson with adequate Lorentz indices. In this paper, we are interested in interactions mediated by massive neutral scalars (Klein-Gordon-type) and spin-1 vector particles (Proca-type), whose momentum-space propagators are

$$
\begin{aligned}
\langle\phi \phi\rangle & =\frac{i}{q^{2}-m_{\phi}^{2}}, \\
\left\langle A_{\mu} A_{\nu}\right\rangle & =\frac{-i}{q^{2}-m_{A}^{2}}\left(\eta_{\mu \nu}-\frac{q_{\mu} q_{\nu}}{m_{A}^{2}}\right) .
\end{aligned}
$$

The sources/currents $J_{1}$ and $J_{2}$, as representatives of the respective vertices, must translate the different possible couplings to the gauge sector: scalar, pseudo-scalar, vector, and pseudo-vector. The vector currents are obtained from the Lagrangian through the Noether method, but, for the bosonic case, we consider only first order in the electromagnetic coupling constant, $e$, since we wish to compute $\mathscr{A}$ to second order in $e$. We will discuss this in more detail in Section 2.2. The other sources/currents are built based on the principle that they should be bilinear in the field and its complex conjugate and reflect the desired symmetry.

Below, we recall some basic properties from spin-1/2 and spin-1 starting from the rest frame where the essential degrees of freedom become apparent and then Lorentz-boosting it to the LAB frame. This will be important when we calculate the NR limits of each source/current and then apply them to extract the interparticle potential. Here, we choose to work the spin-1 in its vector field representation, but, in Section 4, we will discuss it in its less usual tensor field representation $[20,21,37]$.

2.1. Spin-1/2. For the sake of completeness, we present the well-known properties of Dirac fermions. The Dirac equation for the positive-energy spinors is, in momentum-space,

$$
\left[\gamma^{\mu} P_{\mu}-m\right] u(P)=0
$$

with the corresponding equation for the spinor conjugate, $\bar{u} \equiv u^{\dagger} \gamma^{0}$, with the attribution $\bar{u}=\bar{u}\left(P^{\prime}\right)$. By using the gamma matrices in the Dirac representation, the positive-energy spinor can be explicitly written in the NR limit as

$$
u(P) \simeq N_{f}\left(\begin{array}{c}
\xi \\
\frac{\vec{\sigma} \cdot \vec{P}}{2 m} \xi
\end{array}\right),
$$

where $\xi$ are the basic spinors satisfying $\xi_{r}^{\dagger} \xi_{s}=\delta_{r s}$ and $N_{f}$ denotes a normalization constant. Manipulating (5) and its conjugate, we obtain the Gordon decomposition of the vector current:

$$
\bar{u} \gamma^{\mu} u=\frac{p^{\mu}}{m} \bar{u} u+\frac{i}{m} q_{\nu} \bar{u} \Sigma^{\mu \nu} u
$$

expressed in terms of the more convenient variables $p \equiv(P+$ $\left.P^{\prime}\right) / 2$ and $q \equiv P^{\prime}-P$, where we defined the spin matrix as $\Sigma^{\mu \nu} \equiv(i / 4)\left[\gamma^{\mu}, \gamma^{\nu}\right]$. Similar decompositions are also possible for other types of bilinear forms: for example, using (5) and its conjugate, we show that

$$
q_{\mu} \bar{u} \gamma^{\mu} \gamma_{5} u=2 m \bar{u} \gamma_{5} u
$$

The Gordon decomposition of the vector current associated with the Noether theorem (7) yields a first term proportional to the density of the field $\left(\sim|u|^{2}\right)$, while the second one presents a coupling between momentum transfer, $q$, and the spin matrix, $\Sigma^{\mu \nu}$, of the respective representation. This connection will be responsible for the zeroth-order magnetic dipole moment interaction of the lepton with gyromagnetic ratio $g=2$ [38]. Interestingly enough, this simple structure found for the Gordon decomposition for the spin-1/2 will have a perfect analogue in the spin-1 case, if we correctly include a nonminimal coupling; see Section 2.2.

Our interparticle potentials will be derived from the (effective) Lagrangian describing the interactions between the exchanged (scalar or vector) particles and the sources/ currents of the fermionic particle. It reads as follows:

$$
\begin{aligned}
& \mathscr{L}_{\mathrm{int}}^{s=1 / 2} \\
& \quad=\bar{\psi}\left[\left(g_{S}+g_{P S} i \gamma_{5}\right) \phi+\left(g_{V} \gamma^{\mu}+g_{P V} \gamma^{\mu} \gamma_{5}\right) A_{\mu}\right] \psi,
\end{aligned}
$$

where the different couplings are all dimensionless. The scalar $(S)$ and pseudo-scalar (PS) sources are not conserved and, since we are dealing with a massive Dirac field, we also have a nonconserved pseudo-vector $(P V)$ current (see (8)). As pointed out by Boulware [39], the Proca-type field, whenever coupled to a conserved current, leads to a renormalizable model. For this reason, we will consider the coupling between the $P V$ - current and Proca-type field as an effective interaction. The UV divergence does not harm our purposes here, since we are interested in the low-energy regime described by the interparticle potential. Actually, our external (on-shell) sources/currents are tailored in the NR regime, meaning that we are working much below the scale where UV divergences show up. Henceforth we will denote the coupling constant $g_{V} \equiv e$ whenever considering the electromagnetic interaction.

2.2. Spin-1. In the previous subsection, we have revised some basic aspects concerning spin-1/2 particles. Here, we would like to follow up with a similar discussion for massive charged spin-1 particles in order to comparatively study how differently (or not) spin-1/2 and spin-1 sources/currents interact in the NR limit. 
We start off discussing the vector field representation for a massive charged spin-1 particle, whose dynamics can be obtained from the following Lagrangian:

$$
\begin{aligned}
\mathscr{L}_{\text {vec. }}= & -\frac{1}{2} \mathscr{W}_{\mu \nu}^{*} \mathscr{W}^{\mu \nu}+m^{2} W_{\mu}^{*} W^{\mu} \\
& +i e(g-1) W_{\mu}^{*} W_{\nu} F^{\mu \nu},
\end{aligned}
$$

where $m$ is the mass and $\mathscr{W}_{\mu \nu}$ the gauge-covariant fieldstrength, given by $D_{\mu} W_{\nu}-D_{\nu} W_{\mu}$, with $D_{\mu}=\partial_{\mu}+i e A_{\mu}$. The last term in (10) is a nonminimal coupling between the bosonic matter fields and the field-strength of the interaction mediator and its importance lies in the fact that it asserts that $g=2$ in tree-level for the spin-1 particle [40-42] (see also references therein).

The field $W^{\mu}=\left(W^{0}, \vec{W}\right)$ has, in principle, four degrees of freedom and these are reduced through the constraints introduced by the equations of motion, which read

$$
D_{\mu} \mathscr{W}^{\mu \nu}+m^{2} W^{\nu}-i e(g-1) W_{\mu} F^{\mu \nu}=0
$$

with analogue ones for the complex conjugated field. Our goal is to characterize the source/current-source/current interaction and for this, at tree-level, the aforementioned free source/current is to be described by bilinears in the spin-1 matter fields, where the derivatives are the ordinary ones. In other words, since the asymptotic states are the particle states composed only of $W$ fields (we are now taking the free field equations), we have the subsidiary condition $\partial_{\mu} W^{\mu}=0$. This constraint is better seen in momentum-space, where we have $\partial_{\mu}=-i P_{\mu}$, so that

$$
W^{0}=\frac{1}{E} \vec{P} \cdot \vec{W}
$$

where, in order to avoid a cumbersome notation, we have used the same symbol to denote the field in position- and momentum-space, as there is no risk of confusion. This equation relates different components of the vector field and shows that the time-component is proportional to the longitudinal projection of $\vec{W}$ and that $W^{0}$ is first order in velocity, playing the role of a "small" component similar to the spin-1/2 case. It also allows us to write $W^{\mu}$ in its rest frame as $W_{\text {rest }}^{\mu}=N_{W}(0, \vec{\epsilon})$, where $\vec{\epsilon}$ stands for the dimensionless polarization 3-vector in the rest frame of the free particle and $N_{W}$ is a normalization constant.

The next step is to bring the system from rest into motion (i.e., to the LAB frame) via an appropriate Lorentz transformation. In doing so, we obtain

$$
W_{\mathrm{lab}}^{\mu}=N_{W}\left[\frac{1}{m}(\vec{P} \cdot \vec{\epsilon}), \vec{\epsilon}+\frac{1}{m(E+m)}(\vec{P} \cdot \vec{\epsilon}) \vec{P}\right]
$$

and with this two comments are in order:

(i) Equation (12) tells us that, in the rest frame (where $\vec{P}=0$ ), all the information about the vector field is contained in its spatial part. In this frame the only 3 -vector available is the polarization, that is, its spin, which again enforces the vector character of the field. (ii) The normalization constant is given by $N_{W}=1 / \sqrt{2 m}$.

We wish to make some considerations on the vector currents, both global and local, associated with Lagrangian (10). At the global stage, that is, prior to the gauging of $U(1)$ symmetry, the Noether current (in configuration-space) reads

$$
J_{\text {global }}^{\mu}=i e\left(W^{* \mu \nu} W_{v}-W^{\mu \nu} W_{v}^{*}\right) \text {, }
$$

where no covariant derivative is involved, so $W_{\mu \nu} \equiv \partial_{\mu} W_{\nu}-$ $\partial_{\nu} W_{\mu}$. Upon the gauging of the symmetry, and by including the nonminimal coupling $g$-term, the current changes into

$$
\begin{aligned}
J_{V}^{\mu}= & i e\left(\mathscr{W}^{* \mu \nu} W_{\nu}-\mathscr{W}^{\mu \nu} W_{\nu}^{*}\right) \\
& +i e(g-1) \partial_{\nu}\left(W^{\nu} W^{* \mu}-W^{* \nu} W^{\mu}\right) .
\end{aligned}
$$

Now, there is an important remark concerning current conservation: to calculate the current-current potential at the tree-level approximation (one-boson exchange), as we are doing here, we actually only consider the current to the first order in $e$. This amounts to neglecting the terms in the gauge potential present in the current in (15). In other words, to calculate the potential to order $e^{2}$, we consider the current only up to order $e$. Therefore, to the desired order in the coupling constant, the vector current is the globally conserved one, plus the nonminimal $g$-term. At this perturbative level, the current $J_{V}^{\mu}$ is given by the global and nonminimal terms, as displayed in the second line of (16) below:

$$
\begin{aligned}
& \mathscr{L}_{\mathrm{int}}^{s=1}=\left[g_{S} W_{\mu}^{*} W^{\mu}+g_{P S} W_{\mu \nu}^{*} \widetilde{W}^{\mu \nu}\right] \phi+i g_{V}\left[W^{* \mu \nu} W_{\nu}\right. \\
& \left.-W^{\mu \nu} W_{\nu}^{*}+(g-1) \partial_{\nu}\left(W^{\nu} W^{* \mu}-W^{* \nu} W^{\mu}\right)\right] A_{\mu} \\
& \quad+i g_{P V}\left[\widetilde{W}^{* \mu \nu} W_{\nu}-\widetilde{W}^{\mu \nu} W_{\nu}^{*}\right] A_{\mu},
\end{aligned}
$$

where the coupling constants have the following canonical mass dimensions: $\left[g_{S}\right]=-\left[g_{P S}\right]=1$ and $\left[g_{V}\right]=\left[g_{P V}\right]=0$, and the interactions between the sources/currents and the mediating (scalar or vector) bosons are all displayed.

By using the equations of motion and the (free) subsidiary conditions, we may rewrite the vector current in momentumspace as

$$
J_{V}^{\mu}(p, q)=2 e p^{\mu} W_{\nu}^{*} W^{\nu}+i e g q_{\nu} W^{* \alpha}\left(\Sigma_{V}^{\mu \nu}\right)_{\alpha \beta} W^{\beta}
$$

in which $\left(\Sigma_{V}^{\mu \nu}\right)_{\alpha \beta} \equiv i\left(\delta_{\alpha}^{\mu} \delta_{\beta}^{\nu}-\delta_{\alpha}^{\nu} \delta_{\beta}^{\mu}\right)$ stands for the spin matrix in the $(1 / 2,1 / 2)$ representation of the Lorentz group and, as for the $\operatorname{spin}=1 / 2$ case, we have set $g_{V} \equiv e$. In the equation above we left the $g$-factor explicit but in the NR limits we will use its tree-level value, $g=2$. Equation (17) is nothing but the Gordon decomposition for the vector field representation of massive and charged spin- 1 and the similarity with the spin-1/2 case (7) is remarkable. This Gordon decomposition for spin-1 particles was found independently and agrees with the results of [20].

We denote the dual of $W_{\mu \nu}$, as given in the PS- and $P V-$ couplings in (16), by means of $\widetilde{W}_{\mu \nu} \equiv(1 / 2) \epsilon_{\mu \nu \alpha \beta} W^{\alpha \beta}$. We also 
emphasize that the (global) $P V$ - bosonic current is conserved in a topological sense, that is, without making use of either the equations of motion or the symmetries of the Lagrangian. As in the case of spin-1/2, the $S$ - and $P S$ - sources for spin-1 are equally not conserved.

In the next section, we discuss the momentum-space sources/currents for spin-1/2 and spin-1 in the NR limit and calculate the interparticle potentials generated by the exchange of scalar and vector mediating bosons.

\section{Nonrelativistic Currents and Potentials}

Following the indications above, we present below the NR limit of the sources/currents, which may be extracted (in configuration-space) from Lagrangians (9) and (16) as $J \phi$ or $J_{\mu} A^{\mu}$. In the following, the fields are already normalized with $N_{f}=1$ and $N_{W}=1 / \sqrt{2 m}$. From now on, for the sake of clarity, we will adopt the variables $\{p, q\}$ instead of $\left\{P, P^{\prime}\right\}$, so $u(P)=u(p-q / 2)$ and $\bar{u}\left(P^{\prime}\right)=\bar{u}(p+q / 2)$; similar definitions hold for $W_{\mu}(P)$ and $W_{\mu}^{*}\left(P^{\prime}\right)$. We point out that the matter sources/currents developed in this section are for particle 1 , that is, the left vertex in Figure 1. The second source/current may be obtained by taking $\vec{q} \rightarrow-\vec{q}, \vec{p} \rightarrow-\vec{p}$ in the first one.

At this point it is convenient to reinforce the difference between polarization and spin of the particle. In the fermionic case, we will denote the expectation value of its spin, $\sigma_{i}$, by contracting the basic spinors $\xi$ with the Pauli matrices: namely, $\left\langle\sigma_{i}\right\rangle \equiv \xi^{\dagger} \sigma_{i} \xi$. The same idea is applied to the bosonic case, where the spin matrix of the particle is $\left(S_{i}\right)_{j k}=i \epsilon_{i j k}$, with $\Sigma_{i j}=\epsilon_{i j k} S_{k}$, and its expectation value is given by $\left\langle S_{i}\right\rangle \equiv$ $\overrightarrow{\epsilon_{j}^{*}}\left(S_{i}\right)_{j k} \vec{\epsilon}_{k}$.

A remark about our notation: in the process of calculating the amplitude factors, the forms $\xi^{\dagger} \xi \equiv \delta_{i}$ and $\vec{\epsilon}^{*} \cdot \vec{\epsilon} \equiv \delta_{i}$, with $i=1,2$ labeling the particle, will appear frequently. These indicate a possible spin-flip between initial and final states, and we will leave the $\delta$ 's explicit in the final expressions, despite the fact that, in general, low-energy interactions will not induce a change in the spin orientation of the particles involved.

3.1. Scalar (Klein-Gordon Type) Exchange. By means of the relations presented in the previous sections, we can carry out the NR expansion of the $S-$ and $P S$ - of the spin-1 sources. For the $S$-source of spin-1/2 particles, we can use the Dirac spinor (6) and its conjugate to obtain

$$
\begin{gathered}
J_{S(s=1 / 2)}=g_{S}^{1}\left\{\delta_{1}\left[1-\frac{1}{4 m_{1}^{2}}\left(\vec{p}^{2}-\frac{1}{4} \vec{q}^{2}\right)\right]-\frac{i}{4 m_{1}^{2}} \vec{q}\right. \\
\left.\cdot\left(\vec{p} \times\langle\vec{\sigma}\rangle_{1}\right)\right\} .
\end{gathered}
$$

For the $S$-source of the spin-1 particles, we obtain the expression below:

$$
\begin{gathered}
W_{\mu}^{*} W^{\mu}=\frac{g_{S}^{1}}{2 m_{1}}\left\{-\delta_{1}+\frac{1}{2 m_{1}^{2}}\left[\left(\vec{p} \cdot \vec{\epsilon}_{1}\right)\left(\vec{q} \cdot \overrightarrow{\epsilon_{1}^{*}}\right)\right.\right. \\
\left.\left.-\left(\vec{p} \cdot \overrightarrow{\epsilon_{1}^{*}}\right)\left(\vec{q} \cdot \vec{\epsilon}_{1}\right)-\left(\vec{q} \cdot \vec{\epsilon}_{1}\right)\left(\vec{q} \cdot \overrightarrow{\epsilon_{1}^{*}}\right)\right]\right\},
\end{gathered}
$$

which can be written in a more enlightening way if we note that the polarization and momenta 3 -vectors satisfy $-i(\vec{q} \times \vec{p})$. $\langle\vec{S}\rangle=(\vec{p} \cdot \vec{\epsilon})\left(\vec{q} \cdot \vec{\epsilon}^{*}\right)-\left(\vec{p} \cdot \vec{\epsilon}^{*}\right)(\vec{q} \cdot \vec{\epsilon})$. This allows us to set the spin-1 scalar source as

$$
\begin{aligned}
& J_{S(s=1)}=-\frac{g_{S}^{1}}{2 m_{1}}\left\{\delta_{1}\right. \\
& \left.\quad+\frac{1}{2 m_{1}^{2}}\left[i(\vec{q} \times \vec{p}) \cdot\langle\vec{S}\rangle_{1}+\left(\vec{q} \cdot \vec{\epsilon}_{1}\right)\left(\vec{q} \cdot \overrightarrow{\epsilon_{1}^{*}}\right)\right]\right\} .
\end{aligned}
$$

By proceeding analogously, we can find the pseudo-scalar sources listed below:

$$
\begin{aligned}
J_{P S(s=1 / 2)} & =-\frac{i g_{P S}^{1}}{2 m_{1}} \vec{q} \cdot\langle\vec{\sigma}\rangle_{1}, \\
J_{P S(s=1)} & =i g_{P S}^{1} \vec{q} \cdot\langle\vec{S}\rangle_{1},
\end{aligned}
$$

and here we note that both pseudo-scalar sources have the same functional form characterized by the coupling between spin and momentum transfers. In the following, we will present the potentials.

Let us calculate a particular case, namely, that of $S-S$ interaction, for both $s=1$ and $s=1 / 2$ sources. We start off with the fermionic case. As discussed in the Methodology, the general amplitude is given by (2) and, in this case,

$$
\mathscr{A}_{S-S}=\frac{i}{q^{2}-m_{\phi}^{2}} J_{1} J_{2},
$$

where we have used the Klein-Gordon propagator (3) and the momentum assignments of Figure 1:

$$
\begin{aligned}
& J_{1} \equiv g_{S}^{1} \bar{u}\left(p+\frac{q}{2}\right) u\left(p-\frac{q}{2}\right), \\
& J_{2} \equiv g_{S}^{2} \bar{u}\left(-p-\frac{q}{2}\right) u\left(-p+\frac{q}{2}\right) .
\end{aligned}
$$

At this point, we use the fermionic scalar source presented in (18). This scalar source remains unchanged by taking $q \rightarrow$ $-q$ and $p \rightarrow-p$, since it is quadratic in the momenta (it will only be necessary with a change in labels: $1 \rightarrow 2$ ). If we keep terms up to second order in the momenta, the amplitude becomes

$$
\begin{aligned}
\mathscr{A}_{S-S}^{s=1 / 2} \simeq \frac{g_{S}^{1} g_{S}^{2}}{\vec{q}^{2}+m_{\phi}^{2}}\left\{\delta_{1} \delta_{2}[1\right. \\
\left.-\frac{1}{4}\left(\vec{p}^{2}-\frac{\vec{q}^{2}}{4}\right)\left(\frac{1}{m_{1}^{2}}+\frac{1}{m_{2}^{2}}\right)\right]-\frac{i}{4}(\vec{q} \times \vec{p}) \\
\left.\cdot\left[\frac{\delta_{1}\langle\vec{\sigma}\rangle_{2}}{m_{2}^{2}}+\frac{\delta_{2}\langle\vec{\sigma}\rangle_{1}}{m_{1}^{2}}\right]\right\}
\end{aligned}
$$


and the Fourier transformation given in (1) yields the potential:

$$
\begin{aligned}
& V_{S-S}^{s=1 / 2}=-\frac{g_{S}^{1} g_{S}^{2}}{4 \pi} \\
& \quad \cdot \frac{e^{-m_{\phi} r}}{r}\left\{\delta_{1} \delta_{2}\left[1-\frac{1}{4}\left(\vec{p}^{2}+\frac{m_{\phi}^{2}}{4}\right)\left(\frac{1}{m_{1}^{2}}+\frac{1}{m_{2}^{2}}\right)\right]\right. \\
& \left.+\frac{\left(1+m_{\phi} r\right)}{4 r^{2}} \vec{L} \cdot\left(\frac{\delta_{1}\langle\vec{\sigma}\rangle_{2}}{m_{2}^{2}}+\frac{\delta_{2}\langle\vec{\sigma}\rangle_{1}}{m_{1}^{2}}\right)\right\} .
\end{aligned}
$$

As expected, the dominant term is the monopolemonopole given by the Yukawa interaction, $-\delta_{1} \delta_{2} e^{-m_{\phi} r} /$ $(4 \pi r)$. We also obtain second-order corrections to this term in the form of a monopole-dipole with spin-orbit couplings.

The amplitude of the bosonic $S$ - $S$ case presents the same structure as given in (22), but with the sources below:

$$
\begin{aligned}
& J_{1} \equiv g_{S}^{1} W_{\mu}^{*}\left(p+\frac{q}{2}\right) W^{\mu}\left(p-\frac{q}{2}\right), \\
& J_{2} \equiv g_{S}^{2} W_{\mu}^{*}\left(-p-\frac{q}{2}\right) W^{\mu}\left(-p+\frac{q}{2}\right) .
\end{aligned}
$$

We follow the same program; that is, by using the NR limit of the bosonic scalar source (20), we obtain

$$
\begin{aligned}
\mathscr{A}_{S-S}^{s=1} & \simeq \frac{g_{S}^{1} g_{S}^{2}}{4 m_{1} m_{2}\left(\vec{q}^{2}+m_{\phi}^{2}\right)}\left\{\delta_{1} \delta_{2}\right. \\
& +\left[\frac{\delta_{1}}{2 m_{2}^{2}}\left[i(\vec{q} \times \vec{p}) \cdot\langle\vec{S}\rangle_{2}+\left(\vec{q} \cdot \overrightarrow{\epsilon_{2}^{*}}\right)\left(\vec{q} \cdot \vec{\epsilon}_{2}\right)\right]+1\right. \\
& \longleftrightarrow 2]\}
\end{aligned}
$$

and, performing the Fourier integral, we get

$$
\begin{aligned}
V_{S-S}^{s=1} & =-\frac{g_{S}^{1} g_{S}^{2}}{16 \pi m_{1} m_{2}} \frac{e^{-m_{\phi} r}}{r}\left\{\delta_{1} \delta_{2}-\frac{\left(1+m_{\phi} r\right)}{2 r^{2}} \vec{L}\right. \\
& \cdot\left(\frac{\delta_{1}\langle\vec{S}\rangle_{2}}{m_{2}^{2}}+\frac{\delta_{2}\langle\vec{S}\rangle_{1}}{m_{1}^{2}}\right) \\
& +\zeta\left[\frac { \delta _ { 1 } } { 2 m _ { 2 } ^ { 2 } r ^ { 2 } } \left[\delta_{2}\left(1+m_{\phi} r\right)\right.\right. \\
& \left.-\left(\widehat{r} \cdot \vec{\epsilon}_{2}\right)\left(\widehat{r} \cdot \overrightarrow{\epsilon_{2}^{*}}\right)\left(3+3 m_{\phi} r+m_{\phi}^{2} r^{2}\right)\right]+1 \\
& \longleftrightarrow 2]\},
\end{aligned}
$$

where the parameter $\zeta$ in the last term may assume the values $\zeta= \pm 1$; in (28) its value is $\zeta=1$. However, in the particular situation described in Section 4 (a 2-form description of the massive spin-1 particle), the potential (28) also appears, but with $\zeta=-1$.

Here we notice that this potential describes the same interactions as in the fermionic case, namely, the Yukawa factor and a spin-orbit term, but it also displays a "polarizationpolarization" interaction term. This observation will be present in other situations in our comparison between the fermionic and bosonic potentials. Below, we quote the results for the other bosonic potentials:

$$
\begin{aligned}
V_{S-P S}^{s=1} & =\frac{g_{S}^{1} g_{P S}^{2} \delta_{1}}{8 \pi m_{1}} \frac{\left(1+m_{\phi} r\right) e^{-m_{\phi} r}}{r^{2}} \widehat{r} \cdot\langle\vec{S}\rangle_{2} \\
V_{P S-P S}^{s=1} & =\frac{g_{P S}^{1} g_{P S}^{2}}{4 \pi} \\
& \cdot \frac{e^{-m_{\phi} r}}{r}\left\{\left(m_{\phi}^{2}+\frac{3 m_{\phi}}{r}+\frac{3}{r^{2}}\right)\left(\widehat{r} \cdot\langle\vec{S}\rangle_{1}\right)\left(\hat{r} \cdot\langle\vec{S}\rangle_{2}\right)\right. \\
& \left.-\left(\frac{m_{\phi}}{r}+\frac{1}{r^{2}}\right)\langle\vec{S}\rangle_{1} \cdot\langle\vec{S}\rangle_{2}\right\} .
\end{aligned}
$$

The fermionic PS-PS and S-PS potentials have the same functional form as the bosonic ones and, if we neglect a contact term (i.e., a $\delta^{3}(\vec{r})$ term), these results are very similar to those obtained by Moody and Wilczek [25] in the context of fermionic sources exchanging axions. Indeed, they only differ by a mass factor due to the canonical mass dimension of our coupling constants.

3.2. Massive Vector (Proca-Type) Exchange. Firstly, let us focus on the bosonic case, more precisely, on the spin-1 vector current, $J_{V}^{\mu}$. For the $\mu=0$ component in (17), we have, in momentum-space,

$$
\begin{aligned}
& J_{V(s=1)}^{0}=-e_{1}\left[\delta_{1}+\delta_{1}\left(\frac{\vec{p}^{2}}{2 m_{1}^{2}}+\frac{\vec{q}^{2}}{8 m_{1}^{2}}\right)\right]+(g-1) \\
& \quad \cdot \frac{e_{1}}{2 m_{1}^{2}}\left[\left(\vec{p} \cdot \overrightarrow{\epsilon_{1}^{*}}\right)\left(\vec{q} \cdot \vec{\epsilon}_{1}\right)-\left(\vec{q} \cdot \overrightarrow{\epsilon_{1}^{*}}\right)\left(\vec{p} \cdot \vec{\epsilon}_{1}\right)\right. \\
& \left.+\left(\vec{q} \cdot \overrightarrow{\epsilon_{1}^{*}}\right)\left(\vec{q} \cdot \vec{\epsilon}_{1}\right)\right],
\end{aligned}
$$

where the entire second line comes from the $g$-factor correction term. If $g=1$, that line vanishes and we would have an incomplete description of the electromagnetic interaction experienced by the vector boson, as seen from (10). Rearranging the equation above and taking the correct $g=2$-value, we obtain the time-component of the spin-1 vector current in the form

$$
\begin{aligned}
& J_{V(s=1)}^{0} \\
& \qquad \begin{aligned}
= & e_{1} \delta_{1}\left(1+\frac{\vec{p}^{2}}{2 m_{1}^{2}}+\frac{\vec{q}^{2}}{8 m_{1}^{2}}\right) \\
& +\frac{e_{1}}{2 m_{1}^{2}}\left[i(\vec{q} \times \vec{p}) \cdot\langle\vec{S}\rangle_{1}+\left(\vec{q} \cdot \overrightarrow{\epsilon_{1}^{*}}\right)\left(\vec{q} \cdot \vec{\epsilon}_{1}\right)\right],
\end{aligned}
\end{aligned}
$$


while, by taking $\mu=i$ in (17), we get the spatial component as given by

$$
J_{V(s=1)}^{i}=-e_{1}\left[\frac{\delta_{1}}{m_{1}} \vec{p}_{i}+\frac{i}{m_{1}} \epsilon_{i j k} \vec{q}_{j}\left\langle\vec{S}_{k}\right\rangle_{1}\right],
$$

which is very similar with its spin-1/2 counterpart, as we will see in the following.

The spin-1 pseudo-vector current is simpler: according to its expression in (16), we can obtain the following NR limits for $\mu=0$ and $\mu=i$ :

$$
\begin{aligned}
& J_{P V(s=1)}^{0}=\frac{i g_{P V}^{1}}{2 m_{1}} \vec{q} \cdot\langle\vec{S}\rangle_{1}, \\
& J_{P V(s=1)}^{i}=-\frac{g_{P V}^{1}}{2 m_{1}^{2}}\left\{\left[\left(\vec{p}-\frac{1}{2} \vec{q}\right) \cdot \vec{\epsilon}_{1}\right]\left(\vec{q} \times \overrightarrow{\epsilon_{1}^{*}}\right)_{i}\right. \\
& \left.\quad-\left[\left(\vec{p}+\frac{1}{2} \vec{q}\right) \cdot \overrightarrow{\epsilon_{1}^{*}}\right]\left(\vec{q} \times \vec{\epsilon}_{1}\right)_{i}\right\} .
\end{aligned}
$$

We present below the corresponding fermionic currents with the purely vector cases also carrying the electromagnetic coupling constant:

$$
\begin{aligned}
& J_{V(s=1 / 2)}^{0}=e_{1}\left\{\delta_{1}\left[1+\frac{1}{4 m_{1}^{2}}\left(\vec{p}^{2}-\frac{1}{4} \vec{q}^{2}\right)\right]\right. \\
& \left.+\frac{i}{4 m_{1}^{2}}(\vec{q} \times \vec{p}) \cdot\langle\vec{\sigma}\rangle_{1}\right\}, \\
& J_{V(s=1 / 2)}^{i}=e_{1}\left[\frac{\delta_{1}}{m_{1}} \vec{p}_{i}-\frac{i}{2 m_{1}} \epsilon_{i j k} \vec{q}_{j}\left\langle\vec{\sigma}_{k}\right\rangle_{1}\right], \\
& J_{P V(s=1 / 2)}^{0}=\frac{g_{P S}^{1}}{m_{1}} \vec{p} \cdot\langle\vec{\sigma}\rangle_{1}, \\
& J_{P V(s=1 / 2)}^{i}=g_{P V}^{1}\left\{\left[1-\frac{1}{4 m_{1}^{2}}\left(\vec{p}^{2}-\frac{1}{4} \vec{q}^{2}\right)\right]\left\langle\vec{\sigma}_{i}\right\rangle_{1}\right. \\
& \quad-\frac{i \delta_{1}}{4 m_{1}^{2}}(\vec{q} \times \vec{p})_{i} \\
& \left.\quad+\frac{1}{2 m_{1}^{2}}\left[\left(\vec{p} \cdot\langle\vec{\sigma}\rangle_{1}\right) \vec{p}_{i}-\frac{1}{4}\left(\vec{q} \cdot\langle\vec{\sigma}\rangle_{1}\right) \vec{q}_{i}\right]\right\} .
\end{aligned}
$$

Unlike the $V$ - and $P V$ - currents of the spin-1/2 case, the $V$ - and $P V$ - currents of the spin-1 field, as defined in Lagrangian (16) (marked by the $V$ and $P V$ coupling constants), always carry an explicit derivative. At high energies, this extra momentum factor must dominate, but not in the NR limit. This extra derivative affects the way these currents interact with the gauge sector, specially in the case of spin-1. Furthermore, it is interesting to emphasize other differences between the fermionic and bosonic currents above. By comparing the vector currents, we notice essentially an extra term in the bosonic case associated with the contribution of the polarization $(\vec{q} \cdot \vec{\epsilon})(\vec{q} \cdot \vec{\epsilon})$. The $P V$-currents display more remarkable differences. For example, for the $\mu=0$ component ((34) and (38)) we observe that the spin of the fermion couples to the average momentum, $\vec{p}$, while for the bosons the spin couples to the momentum transfer, $\vec{q}$. For the $\mu=i$ components, we have many differences due to the spin terms (see (35) and (39)). These special features will be responsible for the different behaviors of the bosonic and fermionic potentials.

Let us now discuss the potentials involving $V-$ and $P V-$ currents mediated by a Proca-type particle. As already done in the previous subsection, we will exemplify the calculation through a particular configuration and then quote the final results. We choose to calculate one of the simplest cases, the $V-P V$ interaction between $s=1$ currents, since this case contains all the necessary elements to understand the other (lengthier) evaluations.

The amplitude is given by

$$
\mathscr{A}_{V-P V}^{s=1}=i J_{1}^{\mu}\left\langle A_{\mu} A_{\nu}\right\rangle J_{2}^{\nu}=-\frac{1}{\vec{q}^{2}+m_{A}^{2}} J_{1}^{\mu} J_{2 \mu}
$$

where we have used current conservation, $q^{\mu} J_{\mu}=0$, to eliminate the longitudinal contribution of the Proca-type propagator (4). In this particular case $(s=1)$, both currents are conserved. We need therefore to simplify the contraction $J_{1}^{\mu} J_{2 \mu}$ and, according to our assumptions, only the $J_{1}^{0} J_{20}$ piece will contribute in the NR limit, so,

$$
\mathscr{A}_{V-P V}^{s=1} \simeq-i \frac{e_{1} g_{P V}^{2} \delta_{1}}{2 m_{2}} \frac{\vec{q} \cdot\langle\vec{S}\rangle_{2}}{\vec{q}^{2}+m_{A}^{2}},
$$

and we finally calculate the Fourier integral to obtain

$$
V_{V-P V}^{s=1}=-\frac{e_{1} g_{P V}^{2} \delta_{1}}{8 \pi m_{2}} \frac{\left(1+m_{A} r\right) e^{-m_{A} r}}{r^{2}} \widehat{r} \cdot\langle\vec{S}\rangle_{2} .
$$

In a similar way, the case with $s=1 / 2$ currents leads to

$$
\begin{gathered}
V_{V-P V}^{s=1 / 2}=-\frac{e_{1} g_{P V}^{2}}{4 \pi} \frac{e^{-m_{A} r}}{r}\left\{\delta_{1} \vec{p} \cdot\langle\vec{\sigma}\rangle_{2}\left[\frac{1}{m_{1}}+\frac{1}{m_{2}}\right]\right. \\
\left.+\frac{\left(1+m_{A} r\right)}{2 m_{1} r}\left[\langle\vec{\sigma}\rangle_{1} \times\langle\vec{\sigma}\rangle_{2}\right] \cdot \vec{r}\right\}
\end{gathered}
$$

and here the $P V$ - current is not conserved; however, the conservation of the $V$-current ensures that $q_{\mu} q_{\nu}$ term of the Proca propagator drops out.

As we have already seen in the discussion of the currents, the $P V$ - currents of $s=1 / 2$ and $s=1$ exhibit remarkable differences and these explain why the potentials behave so differently. We anticipate that only this fermionic case yields the interaction with the $\vec{p} \cdot\langle\vec{\sigma}\rangle$ dependence and the spinspin contribution of the form $\langle\vec{\sigma}\rangle_{1} \times\langle\vec{\sigma}\rangle_{2}$. In the bosonic case, we have the $\hat{r} \cdot\langle\vec{S}\rangle$ dependence, but this is not exclusive of the $V-P V$ interaction; it also appears in the S-PS bosonic 
potential. The other potentials, $V-V$ and $P V-P V$, are listed below. First, the fermionic ones are as follows:

$$
\begin{aligned}
V_{V-V}^{s=1 / 2}=\frac{e_{1} e_{2}}{4 \pi} \frac{e^{-m_{A} r}}{r}\left\{\delta _ { 1 } \delta _ { 2 } \left[1+\frac{\vec{p}^{2}}{m_{1} m_{2}}\right.\right. \\
\left.+\left(\frac{1}{4 m_{1}^{2}}+\frac{1}{4 m_{2}^{2}}\right)\left(\vec{p}^{2}+\frac{m_{A}^{2}}{4}\right)\right]-\frac{\left(1+m_{A} r\right)}{2 r^{2}} \vec{L} \\
+\left[\left(\frac{\delta_{1}\langle\vec{\sigma}\rangle_{2}}{2 m_{2}^{2}}+\frac{\delta_{2}\langle\vec{\sigma}\rangle_{1}}{2 m_{1}^{2}}\right)\right. \\
\left.+\left(\frac{\delta_{1}\langle\vec{\sigma}\rangle_{2}}{m_{1} m_{2}}+\frac{\delta_{2}\langle\vec{\sigma}\rangle_{1}}{m_{1} m_{2}}\right)\right]+\frac{1}{4 m_{1} m_{2} r^{2}}\langle\vec{\sigma}\rangle_{1} \cdot\langle\vec{\sigma}\rangle_{2} \\
.\left(1+m_{A} r+m_{A}^{2} r^{2}\right)-\frac{1}{4 m_{1} m_{2} r^{2}}\left(\langle\vec{\sigma}\rangle_{1} \cdot \widehat{r}\right)\left(\langle\vec{\sigma}\rangle_{2}\right. \\
\left.. \widehat{r})\left(3+3 m_{A} r+m_{A}^{2} r^{2}\right)\right\},
\end{aligned}
$$

where we can read the electromagnetic potential by setting $m_{A}=0$; here $e_{1}$ and $e_{2}$ stand for the respective (electric) charges of the particles.

For the $P V-P V$ case we obtained

$$
\begin{aligned}
& V_{P V-P V}^{s=1 / 2}=-\frac{g_{P V}^{1} g_{P V}^{2}}{4 \pi} \frac{e^{-m_{A} r}}{r}\left\{\langle\vec{\sigma}\rangle_{1} \cdot\langle\vec{\sigma}\rangle_{2}\right. \\
& \cdot\left[1-\frac{1}{4}\left(\vec{p}^{2}+\frac{m_{A}^{2}}{4}+\frac{m_{A}}{2 r}+\frac{1}{2 r^{2}}\right)\left(\frac{1}{m_{1}^{2}}+\frac{1}{m_{2}^{2}}\right)\right] \\
& +\left(\langle\vec{\sigma}\rangle_{1} \cdot \vec{p}\right)\left(\langle\vec{\sigma}\rangle_{2} \cdot \vec{p}\right)\left(\frac{1}{m_{1} m_{2}}+\frac{1}{2 m_{1}^{2}}+\frac{1}{2 m_{2}^{2}}\right) \\
& +\frac{\left(1+m_{A} r\right)}{4 r^{2}} \vec{L} \cdot\left(\frac{\delta_{2}\langle\vec{\sigma}\rangle_{1}}{m_{2}^{2}}+\frac{\delta_{1}\langle\vec{\sigma}\rangle_{2}}{m_{1}^{2}}\right) \\
& +\frac{1}{8 r^{2}}\left(\langle\vec{\sigma}\rangle_{1} \cdot \widehat{r}\right)\left(\langle\vec{\sigma}\rangle_{2} \cdot \widehat{r}\right)\left(\frac{1}{m_{1}^{2}}+\frac{1}{m_{2}^{2}}\right) \\
& \left.+\left(3+3 m_{A} r+m_{A}^{2} r^{2}\right)\right\}+V_{P V-P V-\text { LONG }}^{s=1 / 2}
\end{aligned}
$$

where

$$
\begin{aligned}
& V_{P V-P V-\mathrm{LONG}}^{s=1 / 2} \equiv \frac{g_{P V}^{1} g_{P V}^{2}}{4 \pi} \frac{e^{-m_{A} r}}{r}\left\{\left(\frac{3}{m_{A}^{2} r^{2}}+\frac{3}{m_{A} r}+1\right)\right. \\
& \cdot\left(\hat{r} \cdot\langle\vec{\sigma}\rangle_{1}\right)\left(\hat{r} \cdot\langle\vec{\sigma}\rangle_{2}\right)-\left(\frac{1}{m_{A}^{2} r^{2}}+\frac{1}{m_{A} r}\right)\langle\vec{\sigma}\rangle_{1} \\
& \left.\cdot\langle\vec{\sigma}\rangle_{2}\right\} .
\end{aligned}
$$

The fermionic $P V-P V$ potential presents some peculiar aspects as an inheritance of the nonconserved $P V$ - currents. First of all, in contrast to other cases, we had to take into account the longitudinal part of the Proca propagator in our calculations. By doing that, we obtain the contribution we have denoted by $V_{P V-P V \text {-LONG }}^{s=1 / 2}$ in (46). For this reason, this potential is not well defined in the massless limit $\left(m_{A} \rightarrow 0\right)$ of the exchanged particle and the excitation of the unphysical spin- 0 component of the massive vector boson $\left(\partial_{\mu} A^{\mu}\right.$ does not decouple any longer) jeopardises the unitarity of the model under study.

In order to give a consistent interpretation and circumvent the unitarity problem, we propose the following physical scenario: the exchanged boson is relatively heavy $\left(m_{1}, m_{2}>\right.$ $\left.m_{A} \gg|\vec{q}|\right)$ and we are bound to work at energy and momentum-transfer levels much below $m_{A}$, so that we are far under the threshold for the excitation of the unphysical longitudinal mode of $A_{\mu}$ as justified by the hierarchy of masses stated above. We highlight two aspects concerning the potential specified by (45) and (46):

(i) We are working in the low momentum-transfer limit and, according to the uncertainty principle, one has the interaction range approximated by $r \sim 1 /|\vec{q}|$. With this and the above hierarchy, we have $r>1 / m_{A}>$ $1 / m_{1}, 1 / m_{2}$, which ensures that we are considering the potential for distances larger than the Compton wavelengths of the $s=1$ particles that act as external currents. This is consistent with the fact that the potential is a meaningful quantity for macroscopically large distances.

(ii) Though our nonconserved pseudo-vector current couples to a massive vector boson, the fact that we are working in a low-energy regime (NR approximation, having in mind heavy $m_{1}$ and $m_{2}$ ) keeps us away from the danger of nonunitarity, which shows up in the high-energy domain $\left(E \gg m_{A}\right)$, so we conclude that the contribution described by (46) is physically meaningful.

The spin- $1 V-V$ potential has the form

$$
\begin{aligned}
V_{V-V}^{s=1} & =\frac{e_{1} e_{2}}{4 \pi} \frac{e^{-m_{A} r}}{r}\left\{\delta _ { 1 } \delta _ { 2 } \left[1+\frac{\vec{p}^{2}}{m_{1} m_{2}}+\left(\frac{1}{m_{1}^{2}}\right.\right.\right. \\
& \left.\left.+\frac{1}{m_{2}^{2}}\right)\left(\frac{\vec{p}^{2}}{2}-\frac{m_{A}^{2}}{8}\right)\right]+\frac{\left(1+m_{A} r\right)}{r^{2}} \vec{L} \\
& +\left[\frac{1}{m_{1} m_{2}}\left(\delta_{1}\langle\vec{S}\rangle_{2}+\delta_{2}\langle\vec{S}\rangle_{1}\right)+\left(\frac{\delta_{1}}{2 m_{2}^{2}}\langle\vec{S}\rangle_{2}\right.\right. \\
& \left.\left.+\frac{\delta_{2}}{2 m_{1}^{2}}\langle\vec{S}\rangle_{1}\right)\right]+\frac{1}{m_{1} m_{2} r^{2}}\left[\langle \vec { S } \rangle _ { 1 } \cdot \langle \vec { S } \rangle _ { 2 } \left(1+m_{A} r\right.\right. \\
& \left.+m_{A}^{2} r^{2}\right)-\left(\hat{r} \cdot\langle\vec{S}\rangle_{1}\right)\left(\hat{r} \cdot\langle\vec{S}\rangle_{2}\right)\left(3+3 m_{A} r\right. \\
& \left.\left.+m_{A}^{2} r^{2}\right)\right]-\zeta\left[\frac { \delta _ { 1 } } { 2 m _ { 2 } ^ { 2 } r ^ { 2 } } \left(\delta_{2}\left(1+m_{A} r\right)\right.\right.
\end{aligned}
$$




$$
\begin{aligned}
& \left.-\left(3+3 m_{A} r+m_{A}^{2} r^{2}\right)\left(\widehat{r} \cdot \vec{\epsilon}_{2}\right)\left(\widehat{r} \cdot \overrightarrow{\epsilon_{2}^{*}}\right)\right)+1 \\
& \longleftrightarrow 2]\},
\end{aligned}
$$

where, as appears in (28) for $V_{S-S}^{s=1}$, we have introduced the parameter $\zeta$. Again, its value in the vector field representation is $\zeta=+1$, whereas $\zeta=-1$ in the case of the tensor field parametrization. We will be more specific about this point in the next section. The electromagnetic potential for the spin-1 charge carriers corresponds to the choice of $m_{A}=0$, with $e_{1}$ and $e_{2}$ denoting the electric charges of the particles. In this case one clearly sees that the electromagnetic Coulomb interaction dominates at leading order for both spin-1/2 and spin-1 currents and, at this level, their respective potentials are indistinguishable.

Finally, the bosonic $P V-P V$ potential is

$$
\begin{aligned}
& V_{P V-P V}^{s=1}=-\frac{g_{P V}^{1} g_{P V}^{2}}{16 \pi m_{1} m_{2}} \\
& \quad \cdot \frac{e^{-m_{A} r}}{r^{3}}\left\{\left(3+3 m_{A} r+m_{A}^{2} r^{2}\right)\left(\hat{r} \cdot\langle\vec{S}\rangle_{1}\right)\left(\hat{r} \cdot\langle\vec{S}\rangle_{2}\right)\right. \\
& \left.\quad-\left(1+m_{A} r\right)\langle\vec{S}\rangle_{1} \cdot\langle\vec{S}\rangle_{2}\right\}
\end{aligned}
$$

and here we recall that the $P V$ - current for $s=1$ is conserved, contrary to what happens with $s=1 / 2$. Therefore, this potential does not present the problems of its fermionic counterpart.

By comparing the results, we notice very similar spindependence in both $V-V$ potentials, such as spin-orbit and spin-spin interactions. However, the bosonic case has additional terms with monopole-monopole and the polarizationpolarization interactions (see the last term in (47)). From the fermionic $P V-P V$ potential, we observe an exclusive spinspin interaction with a $\left(\langle\vec{\sigma}\rangle_{1} \cdot \vec{p}\right)\left(\langle\vec{\sigma}\rangle_{2} \cdot \vec{p}\right)$-dependence. The bosonic case does not present these interactions and it reveals the same functional spin-dependence of the PS-PS potential (30).

To conclude this section, in possession of the potentials we have calculated for spin-1 charged currents/sources, we point out the possibility to apply our results to the study of the recently discovered heavy hyperhydrogen [32] and hyperhelium [33]. To study the details of the spectroscopy of the excited spin- 1 states of $\Lambda$-hypernuclei, a careful analysis of the spin-dependence of the potentials is needed (e.g., see [43]) and, in the particular case of the spin-1 states of the hypernuclei, such an analysis was actually lacking in the literature. Though we are not working out these applications in the present paper-it is out of our scope here-we endeavour to connect our results to the study of the excited spin-1/2 and spin-1 states of heavy hypernuclei. We will report on that elsewhere.

\section{Tensor Field Representation}

We have discussed above the spin-1 particle in terms of the familiar vector field representation, but this is not the only possibility [20,37]. On-shell, a massive charged spin-1 particle may well be described by a complex 2 -form gauge field as given below:

$$
\begin{aligned}
\mathscr{L}_{\text {tens. }}= & -\frac{1}{6} \mathscr{G}^{* \mu \nu \kappa} \mathscr{G}_{\mu \nu \kappa}+\frac{1}{2} m^{2} B^{* \nu \kappa} B_{\nu \kappa} \\
& +i e(g-1) F_{\nu}^{\mu} B_{\mu \kappa} B^{* \nu \kappa},
\end{aligned}
$$

with $\mathscr{G}_{\mu \nu \kappa}=D_{\mu} B_{\nu \kappa}+D_{\kappa} B_{\mu \nu}+D_{\nu} B_{\kappa \mu}$ being the associated gauge-covariant 3-form field-strength. The equations of motion are found to be

$$
\begin{gathered}
D^{\mu} \mathscr{G}_{\mu \nu \kappa}+m^{2} B_{\nu \kappa}+i e(g-1) B_{\mu \kappa} F_{\nu}^{\mu} \\
+i e(g-1) B_{\nu \mu} F_{\kappa}^{\mu}=0 .
\end{gathered}
$$

Following similar arguments as in the Methodology, in the rest frame, we have

$$
\begin{aligned}
& B_{\text {rest }}^{0 i}=0, \\
& B_{\text {rest }}^{i j}=\epsilon_{i j k} \vec{\epsilon}_{k},
\end{aligned}
$$

where $\vec{\epsilon}$ is the polarization 3-vector in the rest frame of the particle. By suitably applying a Lorentz boost we attain the final form for $B^{\mu \nu}$ in the LAB-system:

$$
\begin{aligned}
B^{0 i} & =\frac{N_{B}}{m} \epsilon_{i j k} \vec{\epsilon}_{j} \vec{P}_{k}, \\
B^{i j} & =N_{B}\left\{\epsilon_{i j k} \vec{\epsilon}_{k}\right. \\
& \left.+\frac{1}{m(E+m)}\left[\vec{P}_{i}(\vec{\epsilon} \times \vec{P})_{j}-\vec{P}_{j}(\vec{\epsilon} \times \vec{P})_{i}\right]\right\},
\end{aligned}
$$

where the second term in (53) and the spatial component of (13) can be shown to be analogous: it suffices to consider a specific direction and use the vector identity $\vec{a} \times(\vec{b} \times \vec{c})=$ $(\vec{a} \cdot \vec{c}) \vec{b}-(\vec{a} \cdot \vec{b}) \vec{c}$. The normalization is given by $N_{B}=1 /(2 \sqrt{m})$.

To study the matter sources and currents for this particular representation, we state below the interaction Lagrangian that contemplates all the couplings we are interested in:

$$
\begin{aligned}
& \mathscr{L}_{\mathrm{int}}^{s=1}=\left[g_{S} B_{\mu \nu}^{*} B^{\mu \nu}+g_{P S} B_{\mu \nu}^{*} \widetilde{B}^{\mu \nu}\right] \phi+i g_{V}\left[G^{* \mu \nu \kappa} B_{\nu \kappa}\right. \\
& \left.-G^{\mu \nu \kappa} B_{\nu \kappa}^{*}+(g-1) \partial^{\nu}\left(B_{\nu \alpha} B^{* \mu \alpha}-B_{\nu \alpha}^{*} B^{\mu \alpha}\right)\right] A_{\mu} \\
& +i g_{P V}\left[G^{* \mu \nu \kappa} \widetilde{B}_{\nu \kappa}-G^{\mu \nu \kappa} \widetilde{B}_{\nu \kappa}^{*}\right] A_{\mu},
\end{aligned}
$$

where the coupling constants have the following canonical mass dimensions: $\left[g_{S}\right]=\left[g_{P S}\right]=1$ and $\left[g_{V}\right]=\left[g_{P V}\right]=0$. The 3-form field-strength, $G_{\mu \nu \kappa}$, does not include the gaugecovariant derivative. As previously discussed in connection with the gauge current in the vector field representation, the vector current we present below is the one to order $e$ upon use 
of the subsidiary condition with the ordinary derivative, that is, the free subsidiary condition, which is compatible with our procedure to get the potential to order $e^{2}$.

Following this prescription, we obtained the same result as Delgado-Acosta et al. [20] in terms of the $\{p, q\}$ momentum variables; namely,

$$
\mathscr{J}_{V}^{\mu}(p, q)=2 e p^{\mu} B_{\alpha \beta}^{*} B^{\alpha \beta}+i e g q_{\sigma} B^{* \lambda \kappa}\left(\Sigma_{T}^{\mu \sigma}\right)_{\lambda \kappa, \alpha \beta} B^{\alpha \beta}
$$

where

$$
\begin{aligned}
& \left(\Sigma_{T}^{\kappa \sigma}\right)_{\mu \nu, \alpha \beta}=\frac{1}{2}\left[\eta_{\mu \alpha}\left(\Sigma_{V}^{\kappa \sigma}\right)_{\nu \beta}-\eta_{\nu \alpha}\left(\Sigma_{V}^{\kappa \sigma}\right)_{\mu \beta}\right. \\
& \left.+\eta_{\nu \beta}\left(\Sigma_{V}^{\kappa \sigma}\right)_{\mu \alpha}-\eta_{\mu \beta}\left(\Sigma_{V}^{\kappa \sigma}\right)_{\nu \alpha}\right]
\end{aligned}
$$

is the spin generator of the tensor field representation and, from now on, the symbol $\mathscr{J}$ will denote the momentum-space sources/currents in the tensor field representation.

The NR sources/currents in this representation read

$$
\begin{aligned}
& \mathscr{J}_{S(s=1)}=\frac{g_{S}^{1}}{2 m_{1}}\left\{\delta_{1}+\frac{1}{2 m_{1}^{2}}\left[i(\vec{q} \times \vec{p}) \cdot\langle\vec{S}\rangle_{1}+\delta_{1} \vec{q}^{2}\right.\right. \\
& \left.\left.-\left(\vec{q} \cdot \vec{\epsilon}_{1}\right)\left(\vec{q} \cdot \overrightarrow{\epsilon_{1}^{*}}\right)\right]\right\}, \\
& \mathscr{J}_{P S(s=1)}=-\frac{i g_{P S}^{1}}{2 m_{1}^{2}} \vec{q} \cdot\langle\vec{S}\rangle_{1}, \\
& \mathscr{J}_{V(s=1)}^{0}=e_{1}\left\{\delta_{1}\left(1+\frac{\vec{p}^{2}}{2 m_{1}^{2}}+\frac{\vec{q}^{2}}{8 m_{1}^{2}}\right)\right. \\
& \quad-\frac{e_{1}}{2 m_{1}^{2}}\left[i(\vec{q} \times \vec{p}) \cdot\langle\vec{S}\rangle_{1}+\delta_{1} \vec{q}^{2}\right. \\
& \left.\left.-\left(\vec{q} \cdot \vec{\epsilon}_{1}\right)\left(\vec{q} \cdot \epsilon_{1}^{*}\right)\right]\right\}, \\
& \mathscr{J}_{V(s=1)}^{i}=e_{1}\left[\frac{\delta_{1}}{m_{1}} \vec{p}_{i}+\frac{i}{m_{1}} \epsilon_{i j k} \vec{q}_{j}\left\langle\vec{S}_{k}\right\rangle\right] \\
& \mathscr{J}_{P V(s=1)}^{0}=-\frac{i g_{P V}^{1}}{2 m_{1}} \vec{q} \cdot\langle\vec{S}\rangle_{1}, \\
& \mathcal{O}\left(v^{2}\right) \cdot
\end{aligned}
$$

The sources/currents of the two different spin-1 representations are very similar in the spin sector. Basically, a global sign distinguishes one from the other in every case, but this sign does not interfere in the evaluations of the amplitude. The only exception is the spatial component of the pseudovector current, where we notice some differences in the functional form. Nevertheless, this detail is irrelevant, because its contribution to the $P V-P V$ or $V-P V$ potentials leads to terms of order higher than two in the momenta, which we are currently ignoring in our calculations. For this reason, we refrain from giving the explicit (lengthy) form of $\mathscr{J}_{P V(s=1)}^{i}$ and only indicate its order.
The remarkable differences appear in the polarization sector of the scalar source and time-component of the vector current. We recall that, in the vector field representation ((20) and (32)), we have obtained the following polarizationdependence:

$$
(\vec{q} \cdot \vec{\epsilon})\left(\vec{q} \cdot \overrightarrow{\epsilon^{*}}\right)=\vec{q}^{2}\left(\frac{\vec{q}_{i} \vec{q}_{j}}{\vec{q}^{2}}\right) \vec{\epsilon}_{i} \vec{\epsilon}_{j}^{*}
$$

while in the tensor case (see (57) and (59)), we have

$$
\delta \vec{q}^{2}-(\vec{q} \cdot \vec{\epsilon})(\vec{q} \cdot \vec{\epsilon})=\vec{q}^{2}\left(\delta_{i j}-\frac{\vec{q}_{i} \vec{q}_{j}}{\vec{q}^{2}}\right) \vec{\epsilon}_{i} \vec{\epsilon}_{j}^{*}
$$

According to these results, the two representations differ also due to opposite projections in the contribution coming from the polarization; this could be associated with the particular representation of the spin-1 we are dealing with. Let us be more specific: in order to evaluate the NR limit of these particular cases, we had to take into account the "weak" contributions (those with momenta) of the fields, such as the components $W^{0} \sim \vec{P} \cdot \vec{\epsilon}$ and $B^{0 i} \sim(\vec{P} \times \vec{\epsilon})_{i}$ (see (13) and (53)), respectively, and the analogues present in $\vec{W}$ and $B^{i j}$. These different couplings between momenta and polarization, $\vec{P} \cdot \vec{\epsilon}$ and $\vec{P} \times \vec{\epsilon}$, arise due to the Lorentz boost to the LAB frame and produce the observed longitudinal and transverse projections appearing in (63) and (64) above.

These results ((63) and (64)) raise a question concerning the vector or axial character of the spin-1 particle. In the $W^{\mu}$ case, we are assuming a vector-like particle. In the tensor representation, let us take the components as $B^{0 i}=-B_{0 i} \equiv$ $\vec{X}_{i}$ and $B^{i j}=B_{i j} \equiv \epsilon_{i j k} \vec{Y}_{k}$. On-shell, in the rest frame of the particle, all the degrees of freedom (d.f.) are carried by $\vec{Y}$ (with $\vec{X}=\overrightarrow{0}$ ). Going over to the LAB-system, the tensor representation gives $\vec{X} \sim \vec{P} \times \vec{Y}$. So, in a frame-independent way, the on-shell d.f. are actually described by $\vec{Y}$. The form of the vector current is insensitive to the vector or axial behavior of $\vec{Y}$, as we may conclude by a close inspection of the $\mathcal{g}^{\mu}$ current expressed in terms of $\vec{X}$ and $\vec{Y}$. If $\vec{Y}$ is vector-like, then $\vec{X}$ must be axial, and vice versa. Therefore, we confirm that the minus sign difference highlighted above is always present as a consequence of the choice of representation (vector or tensor), regardless of whether the particle is vector- or axiallike.

At this point, it is instructive to discuss why we do not observe functional differences in all sources/currents, but only a global (numerical or mass) factor. For example, by comparing the spatial component of the vector currents ((33) and (60)), we notice that the first contribution is of order $\mathcal{O}(v)$. One can check that the next correction should be $\mathcal{O}\left(v^{3}\right)$, which we are neglecting in this work. Similar arguments apply to the other cases. Therefore, we could only achieve possible different profiles in these currents if we consider the relativistic corrections to higher orders.

By using the same methodology as in Section 3 it can be shown that the $V-P V$ and $P V-P V$ potentials are identical to the results obtained in the vector field representation ((42) and (48)), while the S-PS and PS-PS only differ by global 
mass factors $\left(1 / 2 m_{2}^{2}\right)$ and $\left(1 / 4 m_{1}^{2} m_{2}^{2}\right)$, respectively, due to the normalization and the different canonical mass dimension of the coupling constant $g_{P S}$ (see (16) and (54)). The other two potentials, $S-S$ and $V-V$, have, respectively, the following profiles:

$$
\begin{aligned}
V_{S-S}^{T}= & \left.V_{S-S}^{s=1}\right|_{\zeta=-1} \\
& +\frac{g_{S}^{1} g_{S}^{2}}{16 \pi m_{1} m_{2}} \frac{e^{-m_{\phi} r}}{r}\left[\delta_{1} \delta_{2} \frac{m_{\phi}^{2}}{2}\left(\frac{1}{m_{1}^{2}}+\frac{1}{m_{2}^{2}}\right)\right], \\
V_{V-V}^{T}= & \left.V_{V-V}^{s=1}\right|_{\zeta=-1} \\
& +\frac{e_{1} e_{2}}{4 \pi} \frac{e^{-m_{A} r}}{r}\left[\delta_{1} \delta_{2} \frac{m_{A}^{2}}{2}\left(\frac{1}{m_{1}^{2}}+\frac{1}{m_{2}^{2}}\right)\right] .
\end{aligned}
$$

The difference between the two representations in both $S-S$ and $V-V$ cases appears as monopole-monopole terms and results in opposite behaviors in the polarization-polarization sector, since $\zeta=+1$ and -1 in the vector and tensor representation, respectively. Actually, these are consequences of what we have seen in the discussion above about the scalar source and time-component of the vector current.

In general, we cannot distinguish between the vector and tensor field representations for the $s=1$ by only considering the spin-dependent sector of the potentials. We emphasize that the differences are suppressed because they only show up in order $\mathcal{O}\left(v^{2}\right)$ in the amplitude. This is so because we are here bound to consider only dipole contributions. If we extend our calculations to also include quadrupole effects, the differences in the $V-V$ potentials for the vector and tensor representation of the spin-1 might become evident, as we could expect from the studies reported in [20]. The consequences of the quadrupole moments of the two representations are interesting to be investigated and we intend to report on that in a forthcoming work [44].

\section{Concluding Remarks}

In this paper, we focused our attention on the special properties of the spin-spin dependence of the source/currentsource/current interaction potential in the nonrelativistic limit for spin-1 and spin-1/2 charged matter particles. Our results indicate some universalities between bosons and fermions when exchanging scalar and vector particles. Essentially, we observe very similar contributions in the spin sector of the $S-S, S-P S, P S-P S$, and $V-V$ potentials. The main differences show up in connection with $P V$ - currents, namely, the $V-P V$ and $P V-P V$ potentials.

We highlight here a result presented in Section 4: the nontrivial consequences of choosing a particular field representation for the spin-1 particle. Even if the vector and tensor representations are equivalent on-shell (and the particles in the source/current are on-shell), the results for the $S-S$ and $V-V$ potentials do differ. The justification was given in the aforementioned section and we claim that, by including higher-order terms in the relativistic corrections, quadrupole moments appear and the differences become explicit.
Furthermore, we do not present a discussion on the bosonic and fermionic (pseudo-)tensor currents exchanging a 2-form gauge field. However, according to preliminary results of a work in progress, it is worthwhile to comment that the case of bosonic sources/currents would yield interesting new interactions involving spin-polarization couplings: for example, $\vec{\epsilon}_{1} \cdot\langle\vec{S}\rangle_{2}$.

To close this report, we point out a question that might be addressed with the help of our results on the comparison between the classes of potentials describing the interaction between spin-1/2 and spin-1 matter sources/currents. For the fermionic case, the electromagnetic potential does not couple to the particle density, as it happens instead for spin- 0 and spin-1 charged matter.

If a charged spin-1 field, $W^{\mu}$, is nonminimally coupled (see (10)) to the electromagnetic field, we have

$$
\begin{aligned}
\partial_{\mu} F^{\mu \nu} & -2 e^{2}\left(W_{\mu}^{*} W^{\mu}\right) A^{\nu} \\
& +e^{2}\left[W^{* v}\left(W_{\mu} A^{\mu}\right)+W^{\nu}\left(W_{\mu}^{*} A^{\mu}\right)\right] \\
= & J_{\text {global }}^{v}+i e \partial_{\mu}\left(W^{\mu} W^{* v}-W^{* \mu} W^{\nu}\right),
\end{aligned}
$$

where $J_{\text {global }}^{\nu}=-i e\left(W_{\mu}^{*} W^{\nu \mu}-W^{* \nu \mu} W_{\mu}\right)$ and $W_{\nu \mu} \equiv \partial_{\nu} W_{\mu}-$ $\partial_{\mu} W_{v}$. The second term of the RHS contributing to $J^{v}$ stems from the nonminimal coupling from (10) and is mandatory to ensure $g=2$ for the charged spin-1 boson.

From (66), two interesting properties of the electromagnetic interactions of spin-1 matter fields are made explicit: the London-type term that couples $A^{\mu}$ to the density of carriers and the new interaction between the photon and the charged boson through their polarization vectors. These special aspects are expected to influence the interparticle potential only if we take loop effects into account.

However, the $e^{2}$-terms in (66) introduce singularities associated with the point-like idealization of spin-1 charged matter particles. The 1962 paper by Dirac [45] and the recent works by Fabbri [46] and Dain [47] address the issue of extensibility in connection with charged particles.

In the present paper, we do not calculate classical field configurations generated by spin-1 currents; instead, we build up interaction potentials by means of a semiclassical calculation, so we do not run into the complications yielded by the singularities mentioned above. It would be nevertheless worthwhile to analyse the details of the connection between particle extension, mass, charge, and spin. The electrodynamics of bosonic carriers seems to suggest that the point-like idealization of charged particles is indeed very restrictive.

We hope to carry on with our studies in this direction and to make use of our results for the spin-1 potentials to get a deeper insight into the understanding of the electrodynamical properties of charged bosonic fields in the classical regime.

\section{Competing Interests}

The authors declare that there is no conflict of interests regarding the publication of this paper. 


\section{Acknowledgments}

The authors would like to thank the National Council for Scientific and Technological Development of Brazil (CNPq), the Coordination for Qualification of Higher Education Personnel (CAPES), the German Service for Academic Exchange (DAAD), and the Fundação Carlos Chagas Filho de Amparo à Pesquisa do Estado do Rio de Janeiro (FAPERJ) for the invaluable financial support.

\section{References}

[1] P. T. Matthews and A. Salam, "The renormalization of meson theories," Reviews of Modern Physics, vol. 23, pp. 311-314, 1951.

[2] A. Salam, "Renormalization of scalar electrodynamics using $\beta$ formalism," Proceedings of the Royal Society of London A, vol. 211, pp. 276-284, 1952.

[3] A. Salam, "Renormalized S-matrix for scalar electrodynamics," Physical Review, vol. 86, no. 5, pp. 731-744, 1952.

[4] A. Komar and A. Salam, "Renormalization problem for vector meson theories," Nuclear Physics B, vol. 21, pp. 624-630, 1960.

[5] A. Salam, "Renormalizability of gauge theories," Physical Review Letters, vol. 127, no. 1, article 331, 1962.

[6] A. Salam, "Renormalizable electrodynamics of vector mesons," Physical Review, vol. 130, no. 3, pp. 1287-1290, 1963.

[7] T. D. Lee and C. N. Yang, "Theory of charged vector mesons interacting with the electromagnetic field," Physical Review, vol. 128, no. 2, pp. 885-898, 1962.

[8] A. Salam and R. Delbourgo, "Renormalizable electrodynamics of scalar and vector mesons. II," Physical Review B, vol. 135, no. 6, article 1398, 1964.

[9] K. H. Tzou, "Covariant electrodynamics of charged mesons of spin one with arbitrary anomalous magnetic moment," Il Nuovo Cimento, vol. 33, no. 2, pp. 286-308, 1964.

[10] H. Aronson, "Spin-1 electrodynamics with an electric quadrupole moment," Physical Review, vol. 186, article 1434, 1969.

[11] G. Velo and D. Zwanzinger, "Noncausality and other defects of interaction Lagrangians for particles with spin one and higher," Physical Review, vol. 188, no. 5, pp. 2218-2222, 1969.

[12] M. Gomez-Bock, M. Mondragón, M. Mühlleitner, M. Spira, and P. M. Zerwas, "Concepts of electroweak symmetry breaking and Higgs physics," http://arxiv.org/abs/0712.2419.

[13] J. F. Nieves and P. B. Pal, "Electromagnetic properties of neutral and charged spin-1 particles," Physical Review D-Particles, Fields, Gravitation and Cosmology, vol. 55, no. 5, pp. 3118-3130, 1997.

[14] A. J. Silenko, "Analysis of wave equations for spin-1particles interacting with an electromagnetic field," http://arxiv.org/abs/ hep-th/0404074.

[15] A. Y. Silenko, "The motion of particle spin in a nonuniform electromagnetic field," Journal of Experimental and Theoretical Physics, vol. 96, no. 5, pp. 775-781, 2003.

[16] A. J. Silenko, "Polarization of spin-1 particles in a uniform magnetic field," European Physical Journal C, vol. 57, no. 3, pp. 595-599, 2008.

[17] A. J. Silenko, "Quantum-mechanical description of spin-1 particles with electric dipole moments," Physical Review D, vol. 87, no. 7, Article ID 073015, 2013.

[18] A. Ross and B. R. Holstein, "Spin effects in the effective quantum field theory of general relativity," Journal of Physics A: Mathematical and Theoretical, vol. 40, p. 6973, 2007.
[19] B. R. Holstein and A. Ross, "Spin effects in long range gravitational scattering," http://arxiv.org/abs/0802.0716.

[20] E. G. Delgado-Acosta, M. Kirchbach, M. Napsuciale, and S. Rodríguez, "Electromagnetic multipole moments of elementary spin-1/2, 1, and 3/2 particles," Physical Review D, vol. 85, no. 11, Article ID 116006, 2012.

[21] E. G. Delgado-Acosta, M. Kirchbach, M. Napsuciale, and S. Rodríguez, "Compton scattering off massive fundamental bosons of pure spin 1," Physical Review D, vol. 87, Article ID 096010, 2013.

[22] M. Napsuciale and S. Goméz-Ávila, "Parity-based formalism for high spin matterfields," http://arxiv.org/abs/1309.0968.

[23] N. E. J. Bjerrum-Bohr, B. R. Holstein, L. Planté, and P. Vanhove, "Graviton-photon scattering," Physical Review D, vol. 91, no. 6, Article ID 064008, 2015.

[24] B. A. Dobrescu and I. Mocioiu, "Spin-dependent macroscopic forces from new particle exchange," Journal of High Energy Physics, vol. 2006, no. 11, article 5, 2006.

[25] J. E. Moody and F. Wilczek, "New macroscopic forces?" Physical Review D, vol. 30, no. 1, pp. 130-138, 1984.

[26] F. A. Ferreira, P. C. Malta, L. P. Ospedal, and J. A. Helayël-Neto, "Topologically massive spin-1 particles and spin-dependent potentials," The European Physical Journal C, vol. 75, aticle 232, 2015.

[27] B. R. Heckel, W. A. Terrano, and E. G. Adelberger, "Limits on exotic long-range spin-spin interactions of electrons," Physical Review Letters, vol. 111, no. 15, Article ID 151802, 2013.

[28] L. Hunter, J. Gordon, S. Peck, D. Ang, and J.-F. Lin, "Using the earth as a polarized electron source to search for long-range spin-spin interactions," Science, vol. 339, no. 6122, pp. 928-932, 2013.

[29] L. R. Hunter and D. G. Ang, "Using geoelectrons to search for velocity-dependent spin-spin interactions," Physical Review Letters, vol. 112, no. 9, Article ID 091803, 2009.

[30] G. Vasilakis, J. M. Brown, T. W. Kornack, and M. V. Romalis, "Limits on new long range nuclear spin-dependent forces set with a K- ${ }^{3}$ He comagnetometer," Physical Review Letters, vol. 103, no. 26, Article ID 261801, 2009.

[31] K. A. Olive, J. F. Arguin, R. M. Barnett et al., "Review of particle physics," Chinese Physics C, vol. 38, no. 9, Article ID 090001, 2014.

[32] M. Agnello, L. Benussi, M. Bertani et al., "Evidence for heavy hyperhydrogen $H_{\Lambda}^{6}$," Physical Review Letters, vol. 108, Article ID 042501, 2012.

[33] T. O. Yamamoto, M. Agnello, Y. Akazawa et al., "Observation of spin-dependent charge symmetry breaking in $\Lambda N$ interaction: $\gamma$-ray spectroscopy of $\mathrm{He}_{\Lambda}^{4}$," Physical Review Letters, vol. 115, no. 22, Article ID 222501, 2015.

[34] F. London and H. London, "The electromagnetic equations of the supraconductor," Proceedings of the Royal Society of London $A$, vol. 149 , no. 866, p. 71, 1935.

[35] M. Maggiore, A Modern Introduction to Quantum Field Theory, Oxford University Press, Oxford, UK, 2005.

[36] A. Accioly, J. Helayël-Neto, F. E. Barone, F. A. Barone, and P. Gaete, "Straightforward prescription for computing the interparticle potential energy related to D-dimensional electromagnetic models," Physical Review D, vol. 90, no. 10, Article ID 105029, 2014.

[37] M. Napsuciale, S. Rodríguez, R. Ferro-Hernández, and S. Gòmez-Ávila, "Spin one matter fields," http://arxiv.org/abs/1509 .07938 . 
[38] L. H. Ryder, Quantum Field Theory, Cambridge University Press, 2nd edition, 1996.

[39] D. G. Boulware, "Renormalizability of massive non-Abelian gauge fields: a functional integral approach," Annals of Physics, vol. 56, pp. 140-171, 1970.

[40] R. Jackiw, "g = 2 as a gauge condition," Physical Review D, vol. 57, no. 4, article 2635, 1998.

[41] S. Ferrara, M. Porrati, and V. L. Telegdi, " $g=2$ As the natural value of the tree-level gyromagnetic ratio of elementary particles," Physical Review D, vol. 46, no. 8, pp. 3529-3537, 1992.

[42] S. Fidelman, K. G. Hayes, K. A. Olive et al., "Review of particle physics," Physics Letters B, vol. 592, no. 1-4, pp. 1-5, 2004.

[43] O. Hashimoto and H. Tamura, "Spectroscopy of $\Lambda$ hypernuclei," Progress in Particle and Nuclear Physics, vol. 57, no. 2, pp. 564653, 2006.

[44] P. C. Malta, L. P. R. Ospedal, K. Veiga, and J. A. Helayél-Neto, "Comparative aspects of spin-dependentinteraction potentials for spin-1/2 and spin-1 matter fields," http://arxiv.org/abs/1510 .03291.

[45] P. A. M. Dirac, "An extensible model of the electron," Proceedings of the Royal Society of London A, vol. 268, no. 1332, pp. 57-67, 1962.

[46] L. Fabbri, "A classical calculation of the leptonic magnetic moment," International Journal of Theoretical Physics, vol. 55, no. 2, pp. 669-677, 2016.

[47] S. Dain, "Bekenstein bounds and inequalities between size, charge, angular momentum, and energy for bodies," Physical Review D, vol. 92, no. 4, Article ID 044033, 2015. 

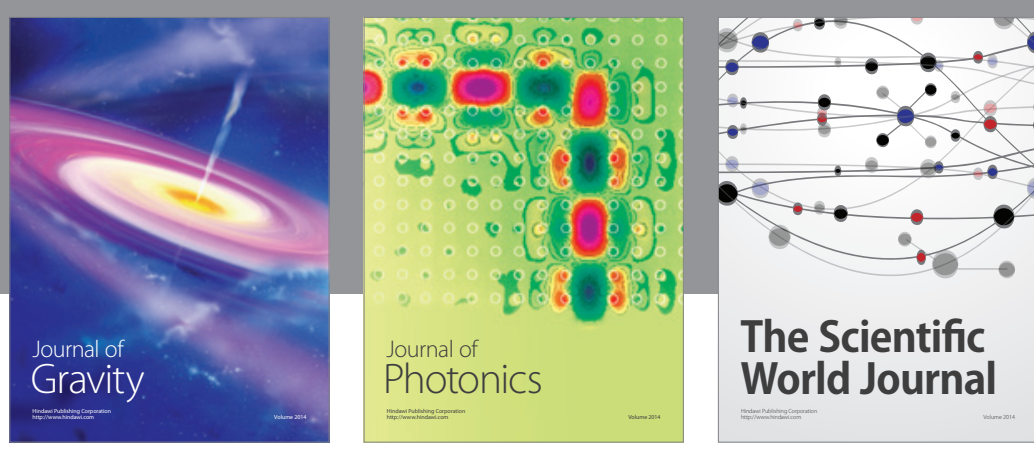

The Scientific World Journal
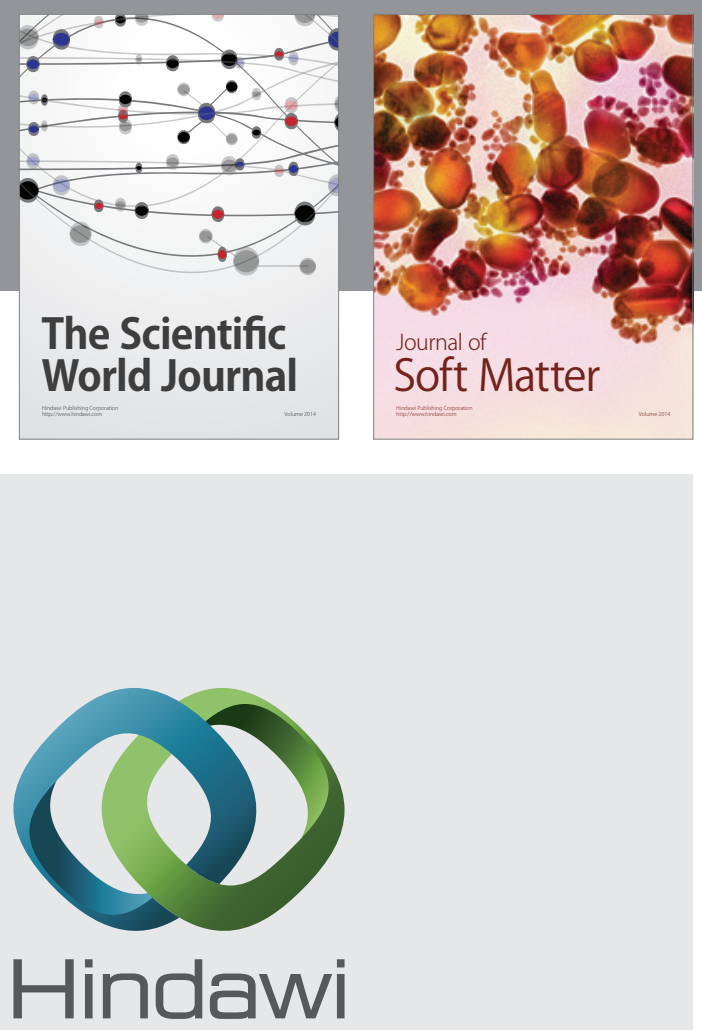

Submit your manuscripts at

http://www.hindawi.com

nternational Journal of

Statistical Mechanics
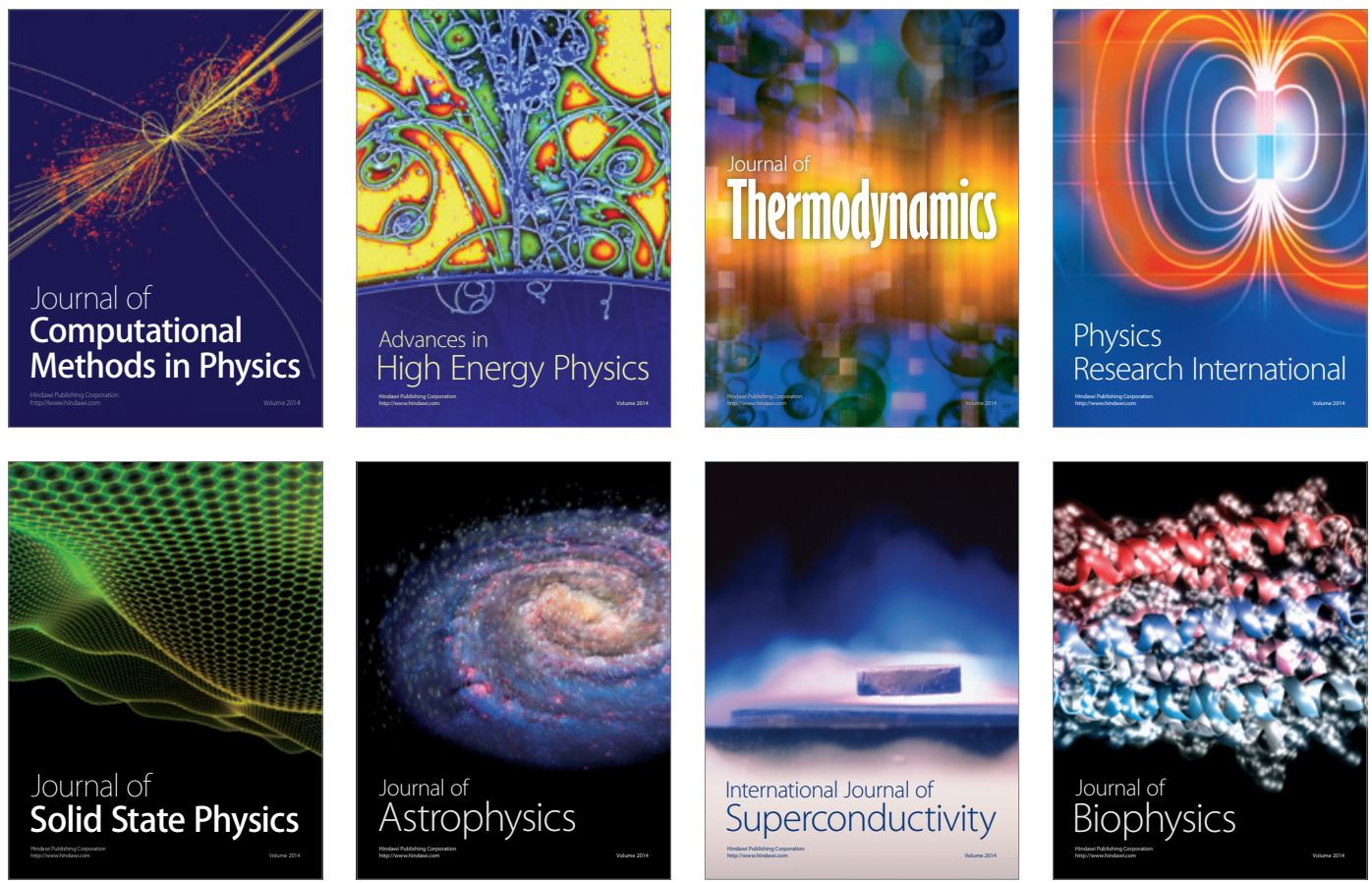
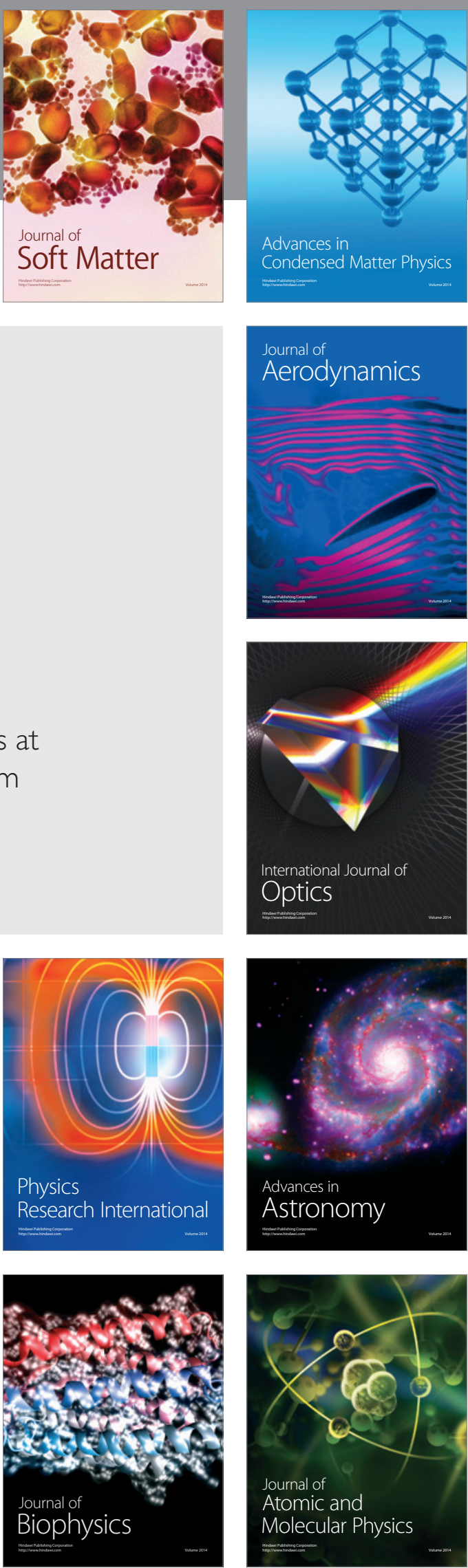